\title{
The occurrence of achromatic transparency
}

\author{
SERGIO CESARE MASIN \\ University of Padua, Padua, Italy \\ and \\ MAMI FUKUDA \\ Keio University, Yokohama, Japan
}

\begin{abstract}
Metelli reported two color conditions for the occurrence of achromatic transparency. However, these conditions apply only to balanced complete transparency. This paper reports a general color condition for the occurrence of both complete (balanced and unbalanced) and partial transparency. Theoretical implications of this finding are discussed.
\end{abstract}

This paper concerns the occurrence of achromatic transparency. In it we show that there is a single color condition for the occurrence of both partial and complete transparency.

Figure 1 represents a transparent disk on a two-part background. Metelli (1974) distinguished between complete and partial transparency. Complete means that the colors of both parts of the background are seen through the transparency, and partial, that the color of only one part of the background is seen through the transparency.

Metelli $(1974,1985)$ identified two color conditions for the occurrence of a special case of complete transparency. In Figure 1, $a$ and $b$ indicate the lightnesses ${ }^{1}$ of the background, and $p$ and $q$, those of the isolated parts of the disk. Metelli (1985) proposed that

$$
p=\alpha a+(1-\alpha) t
$$

and

$$
q=\alpha^{*} b+\left(1-\alpha^{*}\right) t
$$

where $\alpha$ and $\alpha^{*}$ are weights in the real interval $[0,1]$, and $t$ is the overall lightness of the transparent disk. He assumed that $\alpha$ and $\alpha^{*}$ represented the degrees of transparency of the disk on the left and right parts of the background, respectively.

Metelli restricted his discussion to the special case of balanced transparency; that is, when $\alpha=\alpha^{*}$. In this case, Equations 1 and 2 yield

$$
\alpha=(p-q) /(a-b) .
$$

Since $\alpha \in[0,1]$, Equation 3 implies that balanced transparency occurs only when the following relations are satisfied: $p<q$ if $a<b$ (or $p>q$ if $a>b$ ) and $|p-q|<|a-b|$.

Correspondence should be addressed to S. C. Masin, University of Padua, Department of General Psychology, Piazza Capitaniato 3, 35100 Padova, Italy (e-mail: masin@ipdunivx.unipd.it).

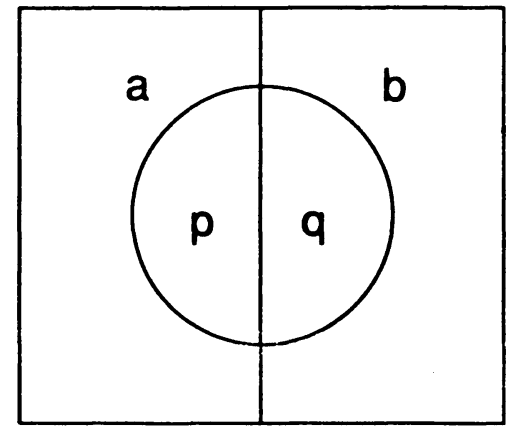

Figure 1. Representation of a transparent disk on a two-part background. The letters indicate the lightnesses of the pattern.

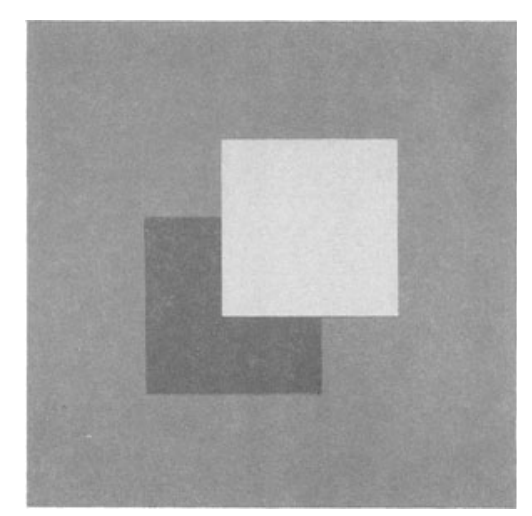

Figure 2. Example of a three-part partially transparent pattern.

Beck, Prazdny, and Ivry (1984) argued that these color conditions are ecologically valid. However, these conditions hold in only one case of only one kind of transparency (i.e., balanced complete transparency). Thus, the conditions for the occurrence of transparency require further investigation. 


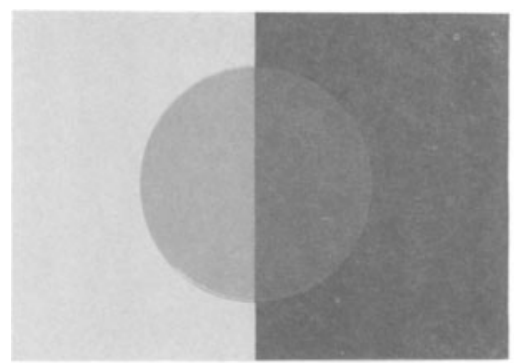
$a>p>q$
$p>q>b$

COMPLETE TRANSPARENCY

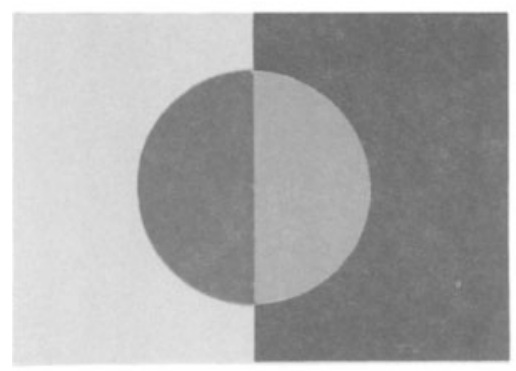
$a>p<q$
$\mathrm{p}<\mathrm{q}>\mathrm{b}$

NO TRANSPARENCY

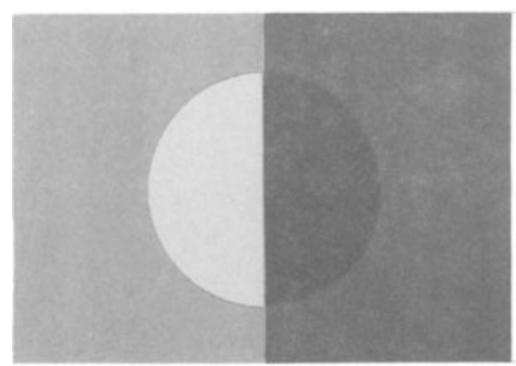

a $<$ p $>$ q $\quad$ p $>$ q $<$ b

NO TRANSPARENCY

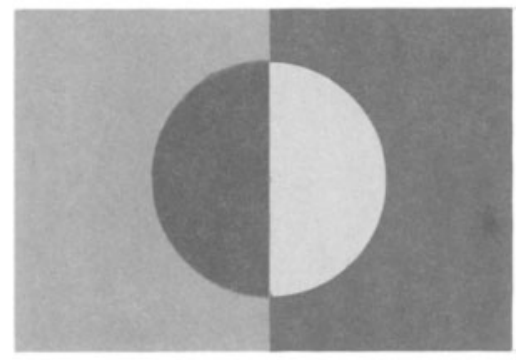

$$
\begin{gathered}
\text { a }>\text { p }<\text { q } \quad \text { p }<\text { q }>\text { b } \\
\text { NO TRANSPARENCY }
\end{gathered}
$$

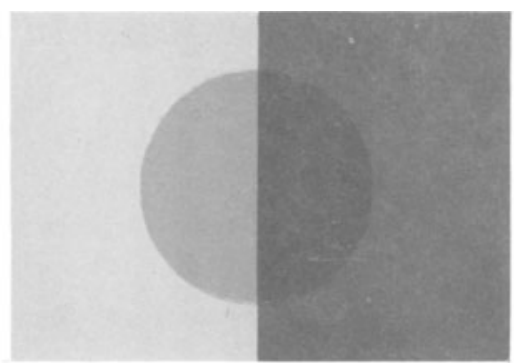
$p>$ q $<$ b

PARTIAL TRANSPARENCY

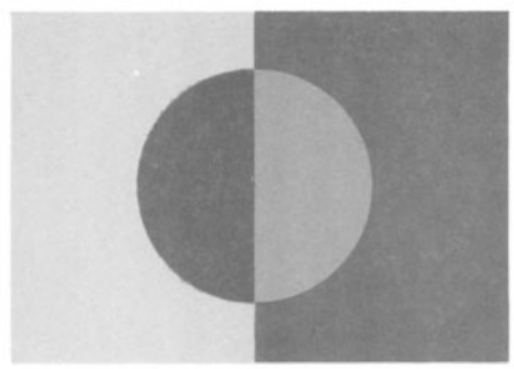

$a>$ p $<$ q

$p<q>b$

NO TRANSPARENCY

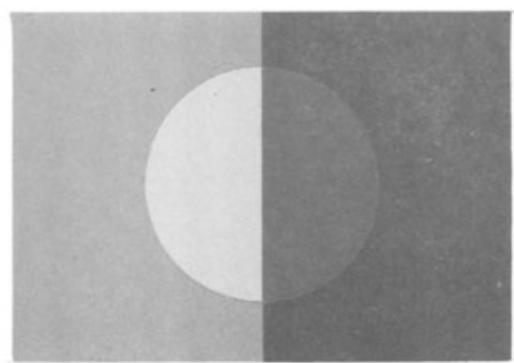

$a<p>q \quad p>q>b$

PARTIAL TRANSPARENCY

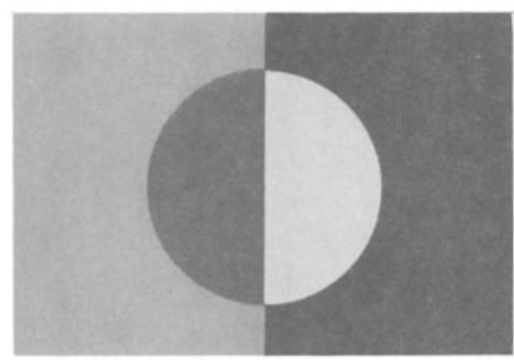

a $>$ p $<$ q $\quad$ p $<$ q $>$ b

NO TRANSPARENCY

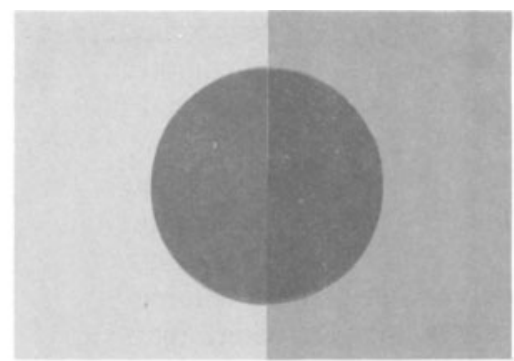

$a>p>q$

$p>$ q $<$ b

PARTIAL TRANSPARENCY

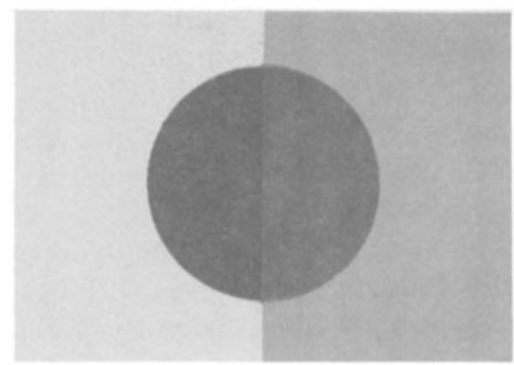

$a>p<q$

$p<q<b$

\section{PARTIAL TRANSPARENCY}

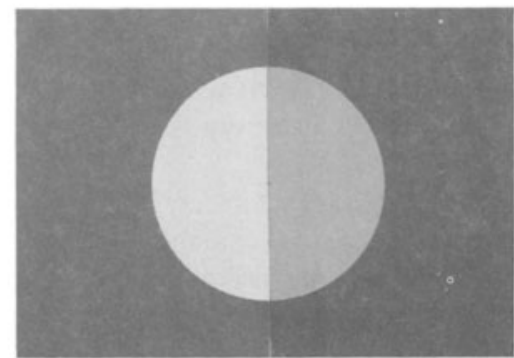

$\mathrm{a}<\mathrm{p}>\mathrm{q}$

$p>q>b$

PARTIAL TRANSPARENCY

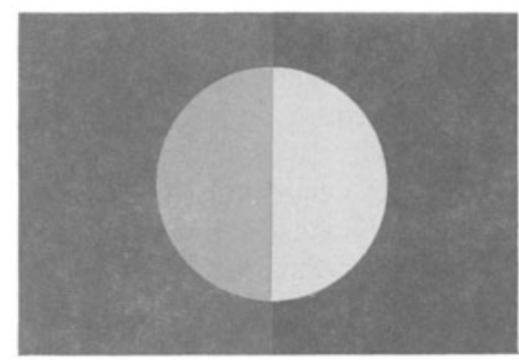

$a<p<q$

$\mathrm{p}<\mathrm{q}>\mathrm{b}$

PARTIAL TRANSPARENCY

Figure 3. Patterns obtained by combining four different lightnesses. The kind of transparency predicted from the order relations between $a, p$, and $q$ and $p, q$, and $b$ is reported below each pattern. 
It is reasonable to assume that if there is a single color condition for the occurrence of both partial and complete transparency, this condition should hold for three-part partially transparent patterns. Figure 2 shows such a pattern, formed by two overlapping squares that appear opaque where they are superimposed on the background and transparent where they are superimposed on each other.

One well-known color condition for the occurrence of partial transparency in three-part patterns is that the lightness of the part where the two transparent surfaces are superimposed on each other is between the lightnesses of the other two opaque parts of the same surfaces (Beck et al., 1984; Metelli, Masin, \& Manganelli, 1981). The existence of additional color conditions for the occurrence of partial transparency in three-part patterns is excluded by Masin's (1991) experimental finding that the lightness of the background is irrelevant for both the occurrence and the judgment of the degree of partial transparency in these patterns. Thus, if a single color condition for the occurrence of both partial and complete transparency should exist, the color condition for the occurrence of partial transparency in three-part patterns should be this single condition.

To see how this condition applies to patterns like that represented in Figure 1, let us first emphasize the prerequisite for the detection of transparency in these patterns by a visual mechanism that processes inputs from retinal contours. Let the perceived disk and the two perceived rectangles with lightnesses $a$ and $b$ correspond to the stimulus disk and the two stimulus rectangles $C, A$, and $B$, respectively. The mechanism needs first to detect $A, B$, and $C$ and then decide whether or not $A, B$, and $C$ intersect. After this prerequisite is met, the following steps are possible.

When $C$ and $A$ intersect, the mechanism checks whether the light intensity corresponding to the intersection of $C$ and $A$ is between the light intensities of the other two parts of $C$ and $A$. When it is, the mechanism decides that there is transparency; otherwise, it decides that there is no transparency. In phenomenal terms, this means that transparency occurs only if the order relation $a<p<q$ (or $a>p>q)$ is satisfied.

Similarly, when $C$ and $B$ intersect, the mechanism checks whether the light intensity corresponding to the intersection of $C$ and $B$ is between the light intensities of the other two parts of $C$ and $B$. When it is, the mechanism decides that there is transparency; otherwise, it decides that there is no transparency. In phenomenal terms, this means that transparency occurs only if the order relation $p<q<b$ (or $p>q>b$ ) is satisfied.

Thus, when the single color condition for the occurrence of both partial and complete transparency is met for both the intersections of $A$ and $C$ and $B$ and $C$, there is complete transparency; when it is met only for one of these intersections, there is partial transparency; and when it is not met for any of these intersections, there is no transparency.

In Figure 3, the reader may check these predictions directly. Each pattern corresponds to one of the 12 dif- ferent combinations of four different values for $a, p, q$, or $b$. (In each pattern, $a>b$. The symmetrical cases with $a<b$ can be obtained by rotating the page $180^{\circ}$.) Below each pattern, the order relations between $a, p$, and $q$, and between $p, q$, and $b$, are reported together with the prediction of transparency-that is, complete, partial, or no transparency. As can be seen, all predictions are confirmed. ${ }^{2}$ Thus, these results indicate that there is a single color condition for the occurrence of both partial and complete transparency.

\section{DISCUSSION}

Fukuda and Masin (1993) have shown experimentally that for fixed $a, q$, and $b$ there is only one value of $p$ for which $\alpha=\alpha^{*}$. That is, in general, transparency is unbalanced. This means that Equation 3 is unjustified. Only separate equations, like Equations 1 and 2 , are justified. ${ }^{3}$

Our finding that transparency occurs only when $a<p<q$ and/or $p<q<b$ suggests other models as alternatives to Equations 1 and 2 . For example, it is possible that the degree of transparency corresponds to the relative position of $p$ in the interval $[a, q]$, or to that of $q$ in the interval $[p, b]$. That is, it is possible that

$$
p=\beta a+(1-\beta) q
$$

or

$$
q=\beta^{*} b+\left(1-\beta^{*}\right) p,
$$

where $\beta=(p-q) /(a-q)$ and $\beta^{*}=(q-p) /(b-p)$ are the degrees of transparency of the disk on the left and right parts of the background, respectively. In this new model, $\beta$ and $\beta^{*}$ vary in the real interval $[0,1]$

Masin $(1984,1991)$ proposed another model. Given the findings reported here, it may be restated as follows. When $a<p<q$,

$$
\begin{aligned}
& \qquad \begin{aligned}
\psi & =w|a-p|+(1-w)(k-|p-q|), \\
\text { and when } p<q & <b \\
\psi^{*} & =w^{*}|q-b|+\left(1-w^{*}\right)(k-|p-q|),
\end{aligned}
\end{aligned}
$$

where $\psi$ and $\psi^{*}$ are the degrees of density (the opposite of transparency) of the disk on the left and right parts of the background, respectively; $w$ and $w^{*}$ are weights in the real interval $[0,1]$; and $k$ is the highest possible degree of density. In this model, $\psi$ and $\psi^{*}$ vary in the real interval $[0, k]$.

No quantitative test has yet been made to decide which of these three models fits the empirical data best.

\section{REFERENCES}

Beck, J., Prazdny, K., \& Ivry, R. (1984). The perception of transparency with achromatic colors. Perception \& Psychophysics, 35, 407-422.

FukudA, M., \& MASIN, S. C. (1993). Perception and judgement of transparency. Manuscript submitted for publication.

KozAKI, T. (1974). The role of background lightness in the apparent transparency (Report No. 7). Yokohama: Keio University, Psychological Laboratory.

MAsin, S. C. (1984). An experimental comparison of three- versus foursurface phenomenal transparency. Perception \& Psychophysics, 35, 325-332.

Masin, S. C. (1987a). Los componentes de la luminosidad subjetiva. Revista Latinoamericana de Psicología, 19, 393-399.

MAsin, S. C. (1987b). The lightness of a transparent surface in Metelli's model of phenomenal transparency. Bulletin of the Psychonomic Society, 25, 263-265.

Masin, S. C. (1991). A weighted-average model of achromatic transparency. Perception \& Psychophysics, 49, 563-571.

Metelli, F. (1974). Achromatic color conditions in the perception of transparency. In R. B. MacLeod \& H. L. Pick (Eds.), Perception: Essays in honor of J. J. Gibson (pp. 95-116). Ithaca, NY: Cornell University Press. 
Metelli, F. (1985). Stimulation and perception of transparency. Psychological Research, 47, 185-202.

Metelli, F., Masin, S. C., \& Manganelli, A. M. (1981). Partial transparency. Atti e Memorie dell'Accademia Patavina di Scienze, Lettere ed Arti, 92, 115-169.

\section{NOTES}

1. Lightness is usually defined as an achromatic color on the blackwhite continuum. According to this definition, lightness is probably a cognitive rather than a perceptual variable, because subjects seem to interpret lightness as "the average of the amounts of black and white" (Masin, 1987a). However, lightness may be defined more simply as the amount of black or the amount of white in an achromatic color.

2. The patterns in Figure 3 are multistable. The probability that two contiguous parts form a surface depends on the lightness difference between them (Kozaki, 1974). Thus, sometimes a rectangle rather than the disk tends to be seen in front. The reader needs to fight against this tendency until the disk is seen in front in order to check directly the predictions for each pattern.

3. From Equations 1 and 2 it follows that

$$
\alpha=(p-t) /(a-t)
$$

and

$$
\alpha^{*}=(q-t) /(b-t) .
$$

Masin (1987b) found experimentally that the judgment of $t$ equals $(p+q) / 2$. It follows that

and

$$
\alpha=(p-q) /(2 a-p-q)
$$

$$
a^{*}=(q-p) /(2 b-p-q) .
$$

(Manuscript received July 12, 1993.) 